\title{
Optimal Angle of Contralateral Oblique View in Cervical Interlaminar Epidural Injection: Safety or Precision?
}

\section{TO THE EDITOR:}

In their article Park et al (1) further analyze the angle to be used for the contralateral oblique (CLO) view. They measure the angle between the mid-sagittal plane and 2 different regions of the dorsal spinal canal: the dorsal spinal canal at the base of the spinous process and the dorsal spinal canal at the base of the lamina. These 2 regions are classified as Zone 1 and Zone 2 respectively (Fig 1) (1). The optimal angle proposed for Zone 1 needles is $10^{\circ}$ degrees $\left(60^{\circ}\right)$ than the angle for Zone 2 needles.

We have previously shown in a prospective study that when using the measured angle approach, the loss of resistance occurs at the ventral interlaminar line (VILL) in the majority of patients. The mean measured angle was $53^{\circ}$ and we had proposed that an angle of $50^{\circ}$ would be a good surrogate. It has been shown that with increasing obliquity the needle tip undergoes posterior translocation $(2,3)$. Thus, using an angle higher than $50^{\circ}$ is likely to place an increasing number of needles on the VILL. The real question is: is increasing obliquity safe?

At what point does the CLO view become a lateral view? As the needle marches laterally, there is a continuum of angles with $90^{\circ}$ for midline needles and ending at around $50^{\circ}$ for needles in Zones 2 and 3 (Fig 2). This variability in angles is a source of ambiguity. What then is the best angle to use? Increasing obliquity makes it possible that the needle tip accesses the epidural space even before the VILL (Fig 3). In this scenario, the consequences of advancing the needle farther, as would be considered appropriate, could be catastrophic. Additionally, at this point, this view is no longer a laminar view, but a hybrid of lateral and laminar views. The laminar appearance will need to be tested prospectively.

A contralateral oblique view enhances the safety of epidural access by crisp visualization of

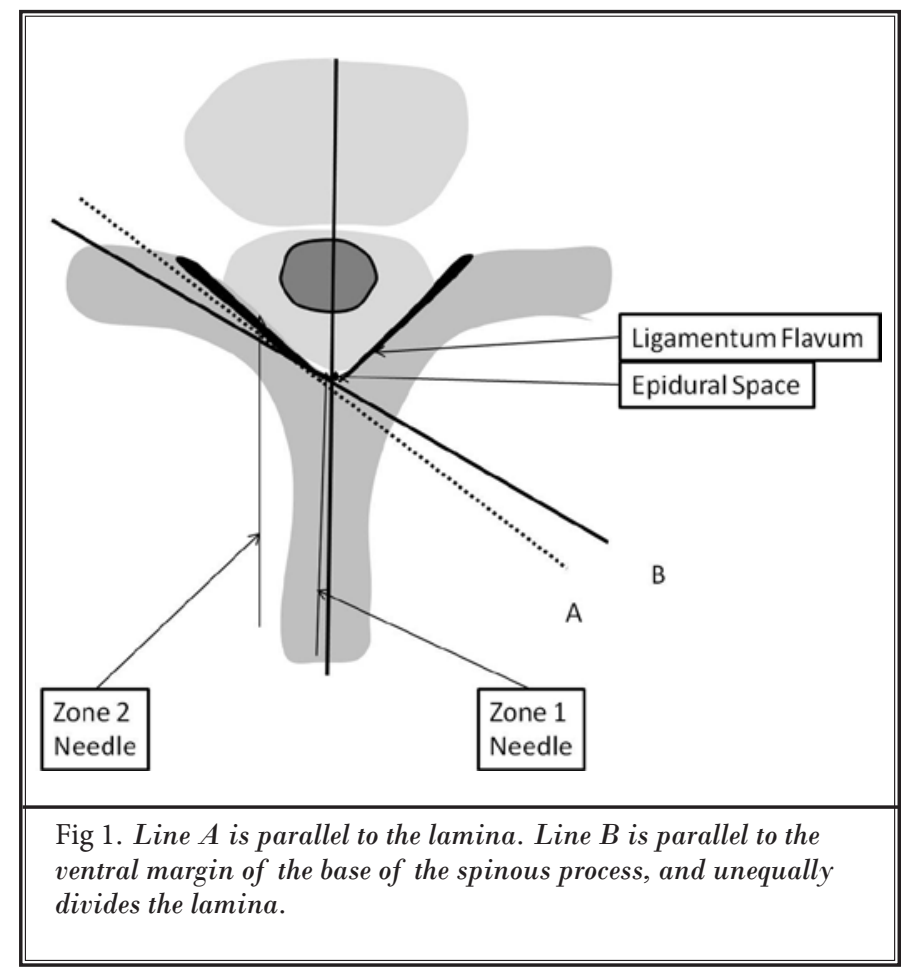

the needle tip as well as providing a landmark at or just beyond which the loss of resistance can be expected. To maintain this safety a standardized approach is needed. Part and parcel of this standardized approach is using a safe obliquity at which the needle will never access the epidural space before encountering the VILL. The measured laminar angle approach guarantees this. A hybrid approach creates ambiguity in terms of the appropriate angle to be used based upon needle location in the anteroposterior (AP) view, and thus diminishes the safety by undermining a standard angle for all positions of the needle tip in the AP view. Additionally, such an obliquity is outside the limits of traditional fluoroscopes. The lateral view makes the most sense with midline needles but often this cannot be used because of several other shortcomings. 

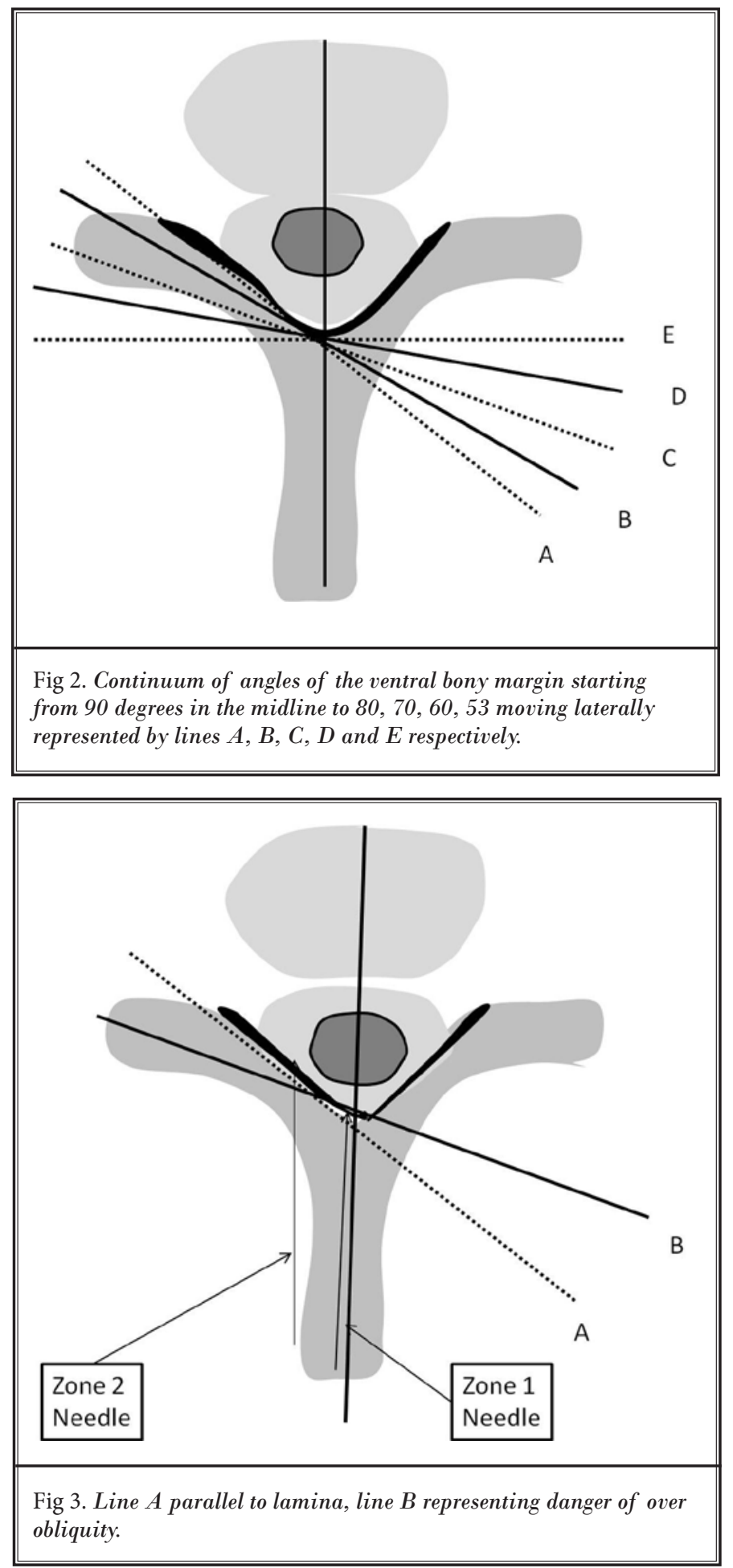

www.painphysicianjournal.com obliquity.
As the authors state, this is not a prospective clinical study, rather retrospective angle measurements on the MRI. A clinical study would be needed to test this hybrid approach against what has already been shown in an experimental design and tested in many clinical practices. A desire for greater precision should not compromise safety.

Jatinder S. Gill, MD

Assistant Professor

Harvard Medical School

Beth Israel Deaconess Medical

Center

Anesthesiology, Critical Care

and Pain Medicine

330 Brookline Avenue

Boston, MA 02215

E-mail:

jsgill@bidmc.harvard.edu

Thomas T. Simopoulos, MD Harvard Medical School

Department of Anesthesiology

Beth Israel Deaconess

One Brookline Place, Ste. 105

Boston MA 02445

tsimopou@bidmc.harvard.edu

\section{References}

1. Park JY, Karm MH, Kim DH, Lee JY, Yun $\mathrm{HJ}$, Suh JH. Optimal angle of contralateral oblique view in cervical interlaminar epidural injection depending on the needle tip position. Pain Physician 2017; 20:E169-E175.

2. Gill J, Aner M, Simopoulos T. Intricacies of the contralateral oblique view for interlaminar epidural access. Pain Med 2013; 14:1265-1266.

3. Gill J, Aner M, Simopoulos T.Gill JS1, Aner M, Nagda JV, Keel JC, Simopoulos TT. Contralateral oblique view is superior to lateral view for interlaminar cervical and cervicothoracic epidural access. Pain Med 2015; 16:68-80. 